\title{
Relation between electromagnetic noise from LED light bulb and its impact on bit error rate performance of DTTB
}

\author{
Ifong $\mathbf{W u}^{\mathrm{a})}$, Hiroki Ohta, Kaoru Gotoh, Shinobu Ishigami, \\ and Yasushi Matsumoto \\ EMC Laboratory, National Institute of Information and Communications Technology \\ 4-2-1 Nukui-Kitamachi, Koganei, Tokyo, 184-8795 Japan \\ a)ifong@nict.go.jp
}

\begin{abstract}
A relation between electromagnetic noise from an LED light bulb and its impact on BER performance of the DTTB is estimated from the APD measurement. First, the APD of the noise is measured with various resolution bandwidths. The characteristic of the APD of the noise is similar to the Gaussian noise when the resolution bandwidth becomes narrow. Then, the BER performance of the DTTB subjected to the noise of the LED light bulb is estimated from the APD result with the resolution bandwidth that is nearly equal to the subcarrier band range of the DTTB. As a result, the BER of the estimated result agrees well with measured results which are subjected to the LED light bulb and the Gaussian noise. Therefore, the effect of the noise from the LED light bulb on the DTTB is similar to the effect of the Gaussian noise.
\end{abstract}

Keywords: noise, LED light bulb, digital terrestrial television broadcasting (DTTB), amplitude probability distribution (APD)

Classification: Electromagnetic compatibility (EMC)

\section{References}

[1] I. Wu, H. Ohta, S. Ishigami, and Y. Matsumoto, "Effects of Noise Radiation from LED Bulbs on communications and Broadcasting Services," IEICE Tech. Rep., EMCJ2011-83, pp. 11-16, 2011.

[2] T. Kasuga, S. Yamada, and H. Inoue, "Measurement of electromagnetic noise radiated from various LED lamps," IEICE Tech. Rep., EMCJ 201166, pp. 29-34, 2011.

[3] S. Kanno and Y. Akiyama, "The mechanism and characteristics of electromagnetic disturbances emitted from LED and fluorescent bulbs," IEICE Tech. Rep., EMCJ 2010-15, pp. 31-34, 2010.

[4] K. Haji, K. Komiya, T. Kobayashi, R. Miyajima, and K. Mikami, "Consideration concerning EMC evaluation of LED application product," IEICE Tech. Rep., EMCJ 2010-80, pp. 57-60, 2011.

[5] P. Wallich, "The lightbulb that really is a better idea [Tools \& Toys]," 
IEEE Spectr., vol. 48, no. 1, pp. 20-22, 2011.

[6] K. Gotoh, Y. Matsumoto, H. Tsutagaya, and S. Kazama, "Evaluation of sensitivity of digital TV receiver subjected to intrasystem interference," Proc. of the 8th Int. Symposium on Electromagnetic Compatibility, Germany, pp. 727-730, Sept. 2008.

[7] K. Gotoh, S. Ishigami, Y. Matsumoto, T. Shinozuka, and Y. Yamanaka, "Electromagnetic disturbance measurement by using amplitude probability distribution for protecting digital wireless communication systems," Journal of National Institute of Information and Communications Technology, Special Issue on EMC, 2006.

[8] M. Sasaki, "Terrestrial digital television broadcasting," NHK STRL Broadcast Technology, no. 20, pp. 14-19, 2004.

[9] L. Wiklundh, "Relation between the amplitude probability distribution of an interfering signal and its impact on digital radio services," IEEE Trans. Electromagn. Compat., vol. 48, no. 3, pp. 537-544, 2006.

[10] Y. Matsumoto, "On the relation between the amplitude probability distribution of noise and bit error probability," IEEE Trans. Electromagn. Compat., vol. 49, no. 4, pp. 940-941, 2007.

\section{Introduction}

Recently, energy saving device such as an LED light bulb is attracting more attention. To improve the efficiency and downsize the power circuit of the LED light bulb, various types of inverter circuits are developed and installed in the LED light bulb [1]. The LED light bulb is expected to become increasingly more widespread in the future. In 2010, poor TV reception happened after installing LED light bulbs at the shopping street in Miyagi Prefecture. The strong electromagnetic noise was found from LED light bulbs in the field survey. This noise of the LED light bulb is generated by the inverter circuit of the LED light bulb and spreads from UHF to VHF bands $[1,2,3,4]$. Many existing broadcasting systems that operate at the same frequency as the noise of the LED light bulb, such as the digital terrestrial television broadcasting (DTTB) and the multimedia broadcasting of Japan may be interrupted by the noise of the LED light bulb. In order to improve the performance of these broadcasting systems, it is necessary to clarify the relation between the noise of the LED light bulb and the broadcasting system. However, it is difficult to evaluate the performance of the broadcasting system owing to the fact that characteristics of the noise of the LED light bulb are not clear and broacasting systems are expensive. For these reasons, it is important to discuss characteristics of the noise leakage from the LED light bulb and evaluate the performance of the broadcasting system more easily. There have been very few studies on effects of noise of the LED light bulb on broadcasting systems [1]. In this paper, an LED light bulb is chosen as an example. This LED light bulb is driven by an inverter circuit and can achieve light output comparable to a 60 -watt incandescent bulb while only using under 100 lumens per watt [5]. First, characteristics of the noise of the LED light bulb are investigated using spectrum analyzer. We also investigate the am- 
plitude probability distribution (APD) [6] of the noise of the LED light bulb. As reported by reference [7], the bit error probability before error-correcting decoding in interfered systems can be estimated from the APD of interfering noise under some assumptions. Then, the relation between the noise from the inverter circuit of the LED light bulb and its impact on bit error rate (BER) performance of the DTTB which is the most popular broadcasting system in Japan is investigated. The BER performance of DTTB is estimated from the APD of the noise of the LED light bulb.

\section{Characteristic of noise of LED light bulb}

To evaluate the relation between the electromagnetic noise from the LED light bulb and its impact on the BER performance of the DTTB, it is necessary to clarify the characteristic of the noise of the LED light bulb. In this section, we investigate the waveform and APD of the noise of the LED light bulb.

\subsection{Waveform of noise of LED light bulb}

To clarify the characteristic of the noise of the LED light bulb, the waveform of the noise of the LED light bulb is evaluated with a spectrum analyzer in zero span. Fig. 1 (a) shows the schematic diagram of the measuring setup. As shown in Fig. 1 (a), the LED light bulb is placed in a transverse electromagnetic (TEM) cell in an anechoic chamber to avoid environmental noise. The TEM cell is used as a receiving equipment to extract noise from the LED light bulb. One of the two ports of the TEM cell on the right side is connected with a $50-\Omega$ terminal. The other port is connected to the spectrum analyzer. Figs. 1 (b) and 1 (c) show results of the waveform of the noise of the LED light bulb. Fig. 1 (b) is the waveform of $100 \mu$ s and Fig. 1 (c) is the waveform of $20 \mathrm{~ms}$. As shown in Fig. 1 (b), the noise from the LED light bulb has a property similar to impulsive noise with a period. The period of the

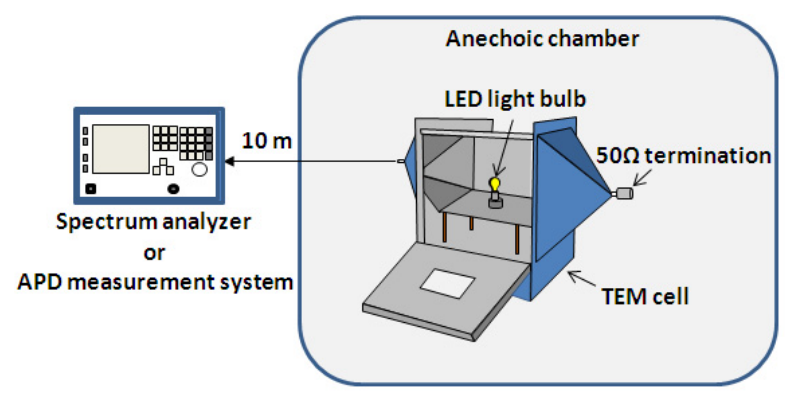

(a) Measurement system of waveform and APD

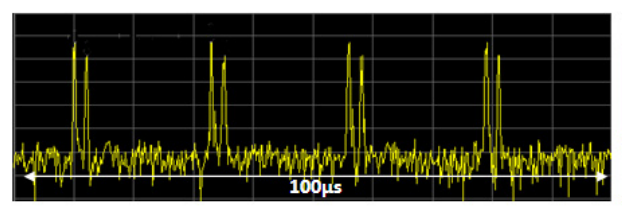

(b) Waveform of noise of LED light bulb $(100 \mu \mathrm{s})$

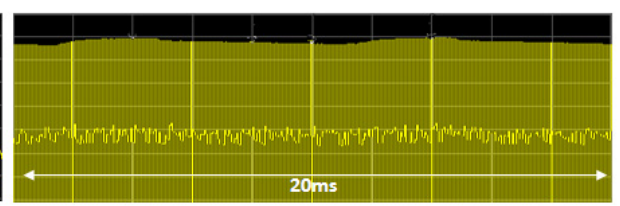

(c) Waveform of noise of LED light bulb (20ms)

Fig. 1. Measurement of waveform of noise of LED light bulb 
noise is $22.7 \mu \mathrm{s}$. This period is almost the same as the switching frequency of the inverter circuit of the LED light bulb. Expanding the time axis, we found that the impulsive noise of this LED light bulb is continuously-occurred as shown in Fig. 1 (c).

\subsection{Amplitude probability distribution (APD)}

APD is a probability of time that the disturbance envelope exceeds the discrete threshold level [7]. The APD is defined as follows:

$$
A P D\left(x_{k}\right)=\sum_{i=1}^{n\left(x_{k}\right)} W_{i}\left(x_{i}\right) / T_{0}
$$

where $W_{i}$ indicate durations in which the disturbance envelope $x(t)$ exceeds the discrete threshold level $x_{k}$ ( $k$ is integer from 1 to $k$ ). The variable $i$ from 1 to $n$ is the number of times that $x(t)$ exceeds $x_{k}$. $T_{0}$ is the total measurement time.

\subsection{APD measurement of noise of LED light bulb}

Measuring APD of interference noise can estimate the amplitude characteristic of the noise. This result can also be directly correlated with performance degradations of interfered communications and broadcasting systems [7]. In this section, the APD of the noise from the LED light bulb is measured. Fig. 1 (a) shows the schematic diagram of the measuring setup. Instead of the spectrum analyzer, the APD measurement system is connected to the TEM cell. Fig. 2 (a) shows results of the APD of the noise of the LED light bulb by various resolution bandwidths of the APD measurement system. The resolution bandwidth of the APD measurement system is measured from $1 \mathrm{kHz}$ to $1 \mathrm{MHz}$. As a result, the noise of the LED light bulb is periodic noise when the resolution bandwidth is $1 \mathrm{MHz}$. We also found that the characteristic of the noise of the LED light bulb is almost the same as the Gaussian noise when the resolution bandwidth decreases. Fig. 2 (b) shows the comparison results between the APD of the LED light bulb and the calculated value of the Gaussian noise. The APD of the Gaussian noise $\left(\mathrm{APD}_{\mathrm{G}}\right)$ is defined as follows:

$$
A P D_{G}(r)=\exp \left(-\frac{r^{2}}{2 \sigma^{2}}\right)
$$

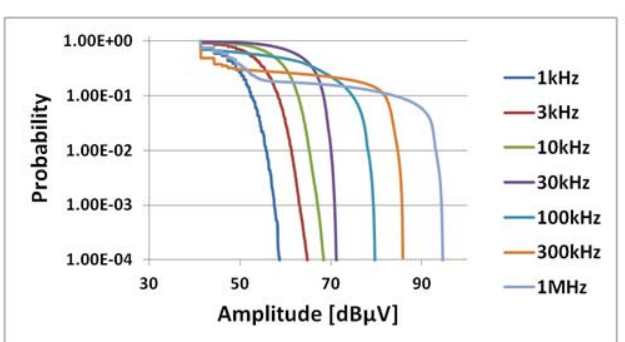

(a) APD of noise from LED light bulb

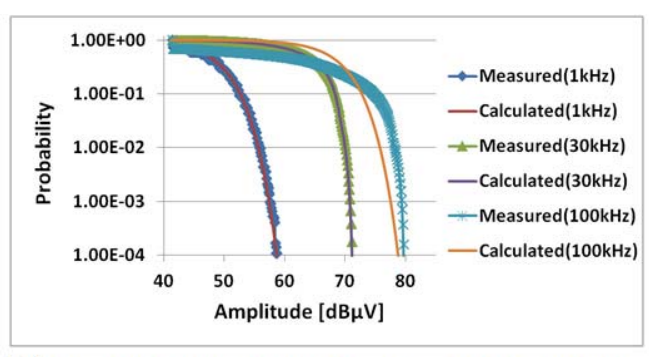

(b) Comparison of measured and calculated results

Fig. 2. APD measurement result of LED light bulb 
where $r$ is the instantaneous voltage of the noise. $\sigma^{2}$ is the averaging power of the noise. As shown in Fig. 2 (b), the APD of the LED light bulb agrees well with the calculated result when the resolution bandwidth is $1 \mathrm{kHz}$. However, the difference is observed between the measured and calculated value when the resolution bandwidth is greater than $100 \mathrm{kHz}$. Therefore, the characteristic of the LED light bulb comes near the Gaussian noise when the resolution bandwidth is below $30 \mathrm{kHz}$.

\section{Relation between noise from LED light bulb and BER}

Broacasting systems are complicate and expensive. Measuring BER characteristic consumes a lot of time. Owing to these reasons, it is necessary to evlaute the relation between noise and its impact on the BER performance more convenient. The APD measurement can be directly correlated with performance degradations of interfered communications and broadcasting systems. Therefore, APD results shown in section 2.3 are useful to utilize in the estimation of performance degradations of the DTTB. In this section, the BER performance of the DTTB subjected to the noise of the LED light bulb is estimated from the APD result. To confirm its reliability, the estimated BER is compared with the measured BER.

\subsection{Digital terrestrial television broadcasting (DTTB)}

The DTTB of Japan is called integrated services digital broadcasting-terrestrial (ISDB-T) using the band segmented transmission-orthogonal frequency division multiplexing (BST-OFDM) [8]. The ISDB-T system uses the UHF band at frequencies between 470 and $710 \mathrm{MHz}$. This bandwidth is divided into fifty channels. Each channel is $5.6 \mathrm{MHz}$ wide and divided into 13 segments. 12 segments of the ISDB-T system are used to broadcast the highdefinition television. 1 segment of the remainder is allocated in a mobile terminal and carries the 1 segment transport stream. The carrier modulation for these 12 segments is 64QAM and for the 1 segment is QPSK. More information about the Japan standard of the DTTB is included in Fig. 3 (a). In this study, we focus on high-definition television (64QAM modulation) to evaluate the relation between the noise from the LED light bulb and its impact on BER performance of the DTTB.

\subsection{BER estimation from APD result}

The BER performance of the DTTB subjected to the noise from the LED light bulb is estimated from the APD result. The BER of the DTTB in terms of APD $\left(B E R_{-} A P D\right)$ for interfered single carrier systems is defined as follows $[6,9,10]$ :

$$
B E R_{-} A P D(A)=\frac{1}{m} A P D\left(\beta A \sqrt{\frac{1}{m}}\right)
$$

where $A$ is rms levels of the DTTB signal. $m$ is number of bits transmitted per a symbol. $\beta$ is a half of the minimum distance between the symbols 
normalized by $A$. In this study, the BER performance of the DTTB is estimated from the APD result with the bandwidth $(1 \mathrm{kHz})$ that is nearly equal to subcarrier band range of the DTTB.

\subsection{BER performance of the DTTB subjected to noise from LED light bulb}

The relation between the noise from the LED light bulb and its impact on BER performance of the DTTB is estimated from the APD without broacasting systems. In this study, we assume power level of the LED light bulb is no different at the measured band. Therefore, the BER of each subcarrier will be affected in the same pattern. To confirm its reliability, the estimated BER is compared with measured BERs which are subjected to the LED light bulb and the Gaussian noise. Fig. 3 (b) shows the schematic of the measurement system of the BER characterastic. Fig. 3(c) shows the comparison results between the estimated and the measured results. As shown in Fig. 3 (c), the estimation result from the APD result agrees well with the measured result which is subjected to the noise of the LED light bulb. The maximum difference between the estimation result and the measured result is less than $1 \mathrm{~dB}$. Therefore, the estimation result from APD is as effective as the BER measurement. We also compare the estimation result with the measured result which is subjected to the Gaussian noise. The BER of these results resultantly agree. Therefore, the effect of the noise from the LED light bulb on DTTB is similar to the effect of the Gaussian noise.

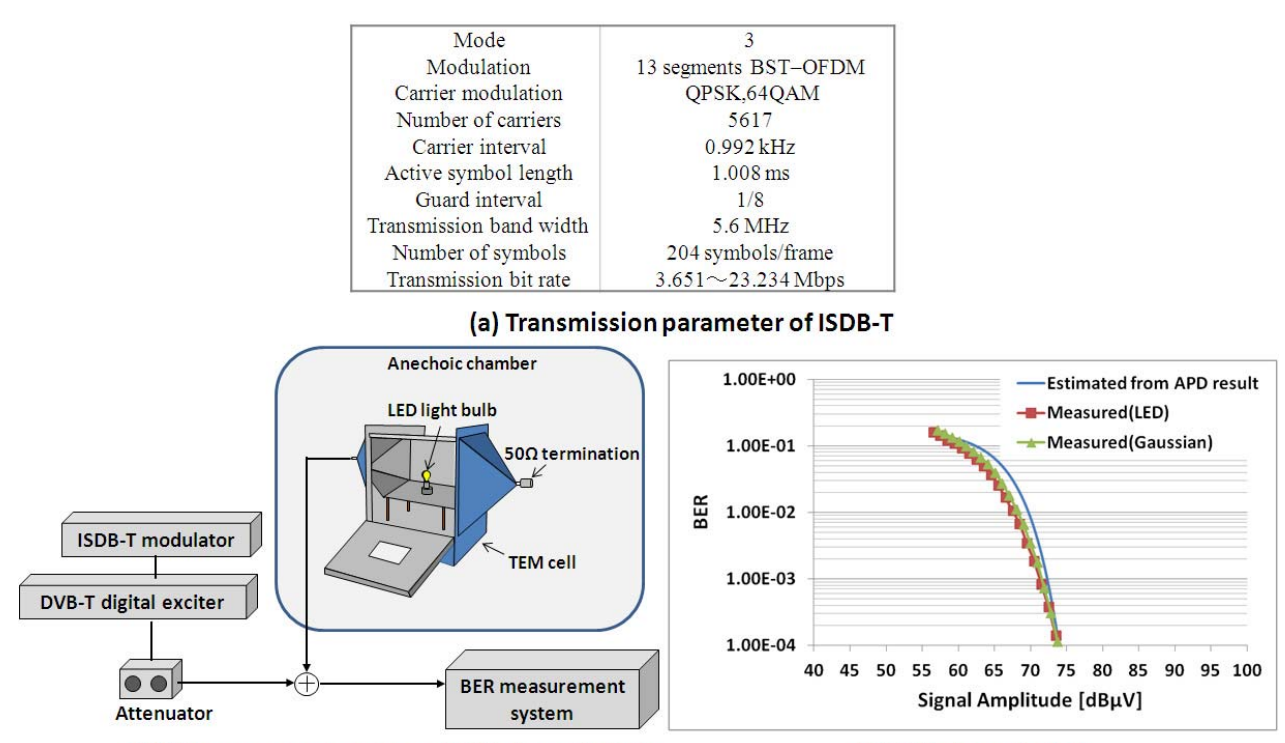

(b) Measurement system of BER characteristic (c) BER characteristic

Fig. 3. BER characteristic of DTTB interfered by noise of LED light bulb

\section{Conclusions}

In this paper, a relation between electromagnetic noise from an LED light 
bulb and its impact on BER performance of the DTTB is investigated without broadcasting systems which are complicate and expensive. First, the APD of the noise of the LED light bulb is measured with various resolution bandwidths. The characteristic of the APD of the LED light bulb appears similar to the impulsive noise when the APD measured with broadband. However, the characteristic of the APD of the LED light bulb turns out to the Gaussian noise when the resolution bandwidth is below $30 \mathrm{kHz}$. Then, the BER performance of the DTTB subjected to the noise of the LED light bulb is estimated from the APD result with the resolution bandwidth $(1 \mathrm{kHz})$ that is nearly equal to the subcarrier band range of the DTTB. As a result, the BER of the estimated result agrees well with the BER measurement which is subjected to the LED light bulb. The estimation method of the APD is as effective as the BER measurement to evaluate the BER performance of the DTTB. We also compare the BER of estimated result with the BER measurement which is subjected to the Gaussian noise. A good agreement between these results is obtained. Therefore, the effect of the noise from the LED light bulb on the DTTB is similar to the effect of the Gaussian noise. 